\title{
Antimicrobial Sensitivity Pattern of Clinical Isolates of Mycobacterium Tuberculosis: A-Retrospective Study from a Reference Laboratory in Pakistan
}

\author{
Tahir Ghafoor ${ }^{1}$, Aamer Ikram $^{2}$, Shahid Ahmad Abbassi ${ }^{3}$, Irfan Ali Mirza ${ }^{4}$, Aamir \\ Hussain $^{5}$, Inam ullah Khan ${ }^{6}$ and Jamal Ahmad ${ }^{7}$ \\ 1, 2, 3, 4, 5, 6 Department of Microbiology, Armed Forces Institute of Pathology (AFIP), Rawalpindi, Pakistan \\ ${ }^{7}$ Department of medicine, PNS Hafeez, Islamabad, Pakistan
}

Correspondence should be addressed to:;

Received date: 8 December 2013; Accepted date: 18 February 2014; Published date: 17 July 2014

Academic Editor: Parvaneh Baghaei

Copyright (C) 2014. Tahir Ghafoor, Aamer Ikram, Shahid Ahmad Abbassi, Irfan Ali Mirza, Aamir Hussain, Inam ullah Khan and Jamal Ahmad. Distributed under Creative Commons CC-BY 3.0

\begin{abstract}
Objective: To determine the sensitivity pattern of first line anti-tuberculosis drugs against clinical isolates of Mycobacterium tuberculosis (MTB) in our settings. Place and duration: Department of Microbiology, Armed Forces Institute of Pathology Rawalpindi, Pakistan, from January 2010 through December 2012. Materials \& Methods: Samples received during the study period were processed on MGIT 960 system for MTB culture and drug susceptibility testing (DST) was performed for first line antituberculosis drugs, namely rifampicin (RIF), isoniazid (INH), streptomycin (STR) and ethambutol (ETH). MTB ATCC 25177 was used as control strain. Results: A total of 4050 samples were tested on MGIT 960 System, out of which $689(17 \%)$ were culture positive. Out of these culture positive cases, $303(44 \%)$ were pansensitive, $52(7.5 \%)$ pan-resistant, $84(12.2 \%)$ sensitive to one drug only, $171(24.8 \%)$ resistant to one drug only and $49(7.1 \%)$ were resistant to 2 drugs other than MDR. $132(19.16 \%)$ cases were multidrug resistant (MDR). Conclusion: Resistance to first line anti-tuberculosis drugs is alarming. Our results highlight the importance of drug susceptibility testing of MTB isolates against first line anti-tuberculosis drugs in an endemic country, so as to properly manage tuberculosis patients.
\end{abstract}

Keywords: Drug susceptibility testing, Multidrug resistant tuberculosis, Mycobacterium tuberculosis, Mycobacteria Growth Indicator Tube System.

Cite this Article as: Tahir Ghafoor, Aamer Ikram, Shahid Ahmad Abbassi, Irfan Ali Mirza, Aamir Hussain, Inam ullah Khan and Jamal Ahmad (2014)," Antimicrobial Sensitivity Pattern of Clinical Isolates of Mycobacterium Tuberculosis: A-Retrospective Study from a Reference Laboratory in Pakistan ", Journal of Virology \& Microbiology , Vol. 2014 (2014), Article ID 385616, DOI: 10.5171/2014. 385616 


\section{Introduction}

Tuberculosis (TB) remains a dreadful infectious disease despite all the advancements in medicine. Adding fuel to the fire is the emergence of MDR TB, which poses a major threat to treatment as resistant cases have a high mortality rate. ${ }^{1}$ World Health Organization (WHO) took an unprecedented step and declared TB as a global emergency in $1993 .^{2}$ As per WHO statistics of 2012, Pakistan stands at fifth position among 22 high TB burden countries. ${ }^{3}$ MDR TB is a real challenge in Pakistan where diagnostic as well as DST facilities for MDR-TB are sparse along with inadequate infection control practices.

There were an estimated 8.7 million incident TB cases worldwide during 2011.3 As per WHO estimates there were 310,000 MDR cases among notified TB patients with pulmonary involvement in 2011. ${ }^{3}$ Almost $60 \%$ of these cases were in China, India and the Russian Federation. ${ }^{3}$ Around $3.7 \%$ of new cases and $20 \%$ of previously treated cases were estimated to have MDR-TB globally. ${ }^{3}$

Pakistan faces a heavy TB toll; according to National Tuberculosis Programme (NTP) Pakistan, 413,450 cases of TB were reported in 2011 with an incidence of $231 / 100,000$ population and prevalence of $364 / 100,000$ population. ${ }^{4}$ In Pakistan, mortality rate with TB is $34 / 100,000.4$ MDR-TB among new treatment cases is $3.2 \%$ and $35 \%$ among retreatment TB cases. ${ }^{4}$ TB treatment success rate is quite encouraging in Pakistan, rising from $77 \%$ in 2001 to $90 \%$ in $2011 .^{4}$

Laboratory capacity to conduct sputum smear microscopy still carries prime importance. The situation is even worst in developing countries like Pakistan, where only 1187 notified laboratories are capable of smear microscopy accounting 0.7 laboratory per 100,000 population, TB culture facility is available in only 12 laboratories accounting 0.3 laboratory per 5 million population, while in whole country only 10 laboratories can perform DST accounting 0.3 laboratory per 5 million population. ${ }^{3}$ According to WHO estimates, during 2011 only 15 of the 22 high TB burden countries met the target of having one microscopy centre per 100,000 population. ${ }^{3}$ In Pakistan, MTB as well as MDR-TB detection modalities like MTBrif assay and MTBDRplus are not yet available, however, gene Xpert is available only at a very few specialized diagnostic/research centers.

Most of the high burden TB countries do not have the recommended capacity of one laboratory per 5 million populations to perform culture and DST. ${ }^{3}$ To ensure the accurate detection of drug resistance for subsequent treatment decisions and to avoid false diagnosis, quality-assured DST is critical. This laboratory based study was aimed to determine the sensitivity pattern of MTB against first line anti-TB drugs in our setup; in fact, a continuation of the previous studies for updating time to time local MTB drug resistance trends for guiding clinicians.

\section{Materials \& Methods}

This retrospective study was carried out in the Department of Microbiology, Armed Forces Institute of Pathology (AFIP), Rawalpindi from January 2010 through December 2012. This laboratory receives samples from tertiary care military hospitals of Rawalpindi and civil hospitals of the surrounding areas.

Various clinical samples were received and dealt according to protocol during the study period. Repeat samples from the same patient, blood samples, bone marrow, urine, stool and improper samples like saliva and pus swabs were excluded from the study. Samples except sterile body fluids were subjected to standard $\mathrm{N}$-acetyl-L-cystine (NALC), $\mathrm{NaOH}$ digestion-decontamination method as described by Kent and Kubica. ${ }^{5}$ Ziehl-Neelsen (ZN) acid fast staining was done while the remaining suspension was inoculated for 42 days on MGIT 960 System as per recommended protocol for the

Tahir Ghafoor, Aamer Ikram, Shahid Ahmad Abbassi, Irfan Ali Mirza, Aamir Hussain, Inam ullah Khan and Jamal Ahmad (2014), Journal of Virology \& Microbiology, DOI: 10.5171/2014. 385616 
method. ${ }^{6}$ Growth yielded on MGIT 960 System was identified as MTB by commercially available immunochromatographic kit (BD MGITTM TBc identification test). DST against first line anti-tuberculosis drugs (RIF, INH, STR and ETH) was performed on all the culture positive isolates by using MGIT 960 System.

Lyophilized drugs (BACTEC MGIT 960 SIRE kit, Becton Dickinson, Baltimore, MD) were dissolved in diluents as per manufacturer's instructions. From the dissolved drug solutions, $100 \mu \mathrm{l}$ was pipetted into a $7.0 \mathrm{ml}$ MGIT 960 tube. The final drug concentrations used were $1.0 \mu \mathrm{g} / \mathrm{ml}$ for STR; $0.1 \mu \mathrm{g} / \mathrm{ml}$ for INH; $5.0 \mu \mathrm{g} / \mathrm{ml}$ for ETH and $1.0 \mu \mathrm{g} / \mathrm{ml}$ for RIF. Results for the sensitivity or resistance to first line anti-TB drugs were interpreted as per standard procedure and were analyzed by using SPSS version 17. MTB ATCC 25177 was used as positive control, while an uninoculated MGIT tube was used as negative control.

\section{Results}

During the 3 years study period, total 4128 samples were received at Microbiology Department of AFIP. 78 samples were contaminated and excluded. Among the remaining 4050 samples, 695 yielded mycobacterial growth including MTB complex (689) and non-tuberculous mycobacteria (6). $388 \quad(56.3 \%)$ culture positive samples were from male patients, while 301 (43.7\%) from female patients, with male to female ratio of $1: 1.3$.

The 3-years data for total number as well as type of samples during the period are shown in table 1 and the resistant patterns are shown in table 2 . Out of total 4050 samples, $689(17 \%)$ were culture positive. Out of these culture positives, 578 (83.9\%) were pulmonary and $111(16.1 \%)$ were extra pulmonary isolates. Out of the culture positives, 303(44\%) were pan-sensitive to all four first line drugs, while 386 (56\%) showed resistance to one or more first line drugs. 52 (7.5\%) isolates were pan-resistant, which were also counted as MDR as per definition of MDR-TB. 84 (12.2\%) isolates turned out to be sensitive to only one drug. Out of these 84 isolates sensitive to only one drug, 50 (59.5\%) were MDR. 171 (24.8\%) isolates were resistant to only one drug, 132 (19.16\%) were MDR (resistant to only RIF + INH) and $49(7.1 \%)$ were resistant to 2 drugs other than MDR, as shown in fig 1.

Mono resistance to INH was found in $13.8 \%$ isolates, RIF 2.6\%, ETH 2.3\% and STR 6.1\% isolates. Overall resistant to STR, INH, RIF and ETH was $27.4 \%, 32.8 \%, 21.2 \%$ and $20.9 \%$ respectively.

Table1: Specimen-wise MTB isolates $(n=689)$

\begin{tabular}{|l|l|}
\hline Specimen & $\mathrm{n}(\%)$ \\
\hline Sputum & $417(60.5 \%)$ \\
\hline EB washings & $161(23.4 \%)$ \\
\hline Tissue & $39(5.7 \%)$ \\
\hline Fluid & $39(5.7 \%)$ \\
\hline Pus & $33(4.8 \%)$ \\
\hline
\end{tabular}

Tahir Ghafoor, Aamer Ikram, Shahid Ahmad Abbassi, Irfan Ali Mirza, Aamir Hussain, Inam ullah Khan and Jamal Ahmad (2014), Journal of Virology \& Microbiology, DOI: 10.5171/2014. 385616 
Table 2: Sensitivity pattern of clinical MTB isolates $(n=689)$

\begin{tabular}{|c|c|c|c|c|c|c|c|c|c|c|c|c|}
\hline \multirow[t]{2}{*}{ Year } & \multicolumn{4}{|c|}{$\begin{array}{c}\text { Mono sensitive } \\
84(12.2 \%)\end{array}$} & \multicolumn{4}{|c|}{$\begin{array}{c}\text { Mono resistant } \\
\text { 171(24.8\%) }\end{array}$} & \multirow{2}{*}{$\begin{array}{c}\text { Pan- } \\
\text { sensitiv } \\
\text { e } \\
(\%)\end{array}$} & \multirow{2}{*}{$\begin{array}{c}\text { Pan- } \\
\text { resista } \\
\text { nt } \\
(\%)\end{array}$} & \multirow{2}{*}{$\begin{array}{c}\text { Total } \\
\text { MDR- } \\
\text { TB } \\
(\%)\end{array}$} & \multirow{2}{*}{$\begin{array}{c}\text { Resista } \\
\text { nt to } 2 \\
\text { drugs } \\
\text { other } \\
\text { than } \\
\text { MDR } \\
(\%)\end{array}$} \\
\hline & $\begin{array}{l}\text { STR } \\
(\%)\end{array}$ & $\begin{array}{l}\text { INH } \\
(\%)\end{array}$ & $\begin{array}{l}\text { RIF } \\
(\%)\end{array}$ & $\begin{array}{l}\text { ETH } \\
(\%)\end{array}$ & $\begin{array}{l}\text { STR } \\
(\%)\end{array}$ & $\begin{array}{l}\text { INH } \\
(\%)\end{array}$ & $\begin{array}{l}\text { RIF } \\
(\%)\end{array}$ & $\begin{array}{l}\text { ETH } \\
(\%)\end{array}$ & & & & \\
\hline 2010 & 3 & 3 & 9 & 15 & 11 & 49 & 8 & 10 & 95 & 14 & 53 & 16 \\
\hline 2011 & 6 & 1 & 16 & 22 & 16 & 38 & 8 & 6 & 124 & 23 & 57 & 21 \\
\hline 2012 & 2 & 2 & 3 & 2 & 15 & 8 & 2 & - & 84 & 15 & 22 & 12 \\
\hline Total & $\begin{array}{l}11 \\
(1.6 \\
)\end{array}$ & $\begin{array}{l}6 \\
60.8 \\
)\end{array}$ & $\begin{array}{l}28 \\
(4.1 \\
)\end{array}$ & $\begin{array}{l}39 \\
(5.7)\end{array}$ & $\begin{array}{l}42 \\
(6.1 \\
)\end{array}$ & $\begin{array}{l}95 \\
(13.8 \\
)\end{array}$ & $\begin{array}{l}18 \\
(2.6 \\
)\end{array}$ & $\begin{array}{l}16 \\
(2.3 \\
)\end{array}$ & $\begin{array}{l}303 \\
(44)\end{array}$ & $\begin{array}{l}52 \\
(7.5)\end{array}$ & $\begin{array}{l}132 \\
(19.16 \\
)\end{array}$ & $\begin{array}{l}49 \\
(7.1)\end{array}$ \\
\hline
\end{tabular}

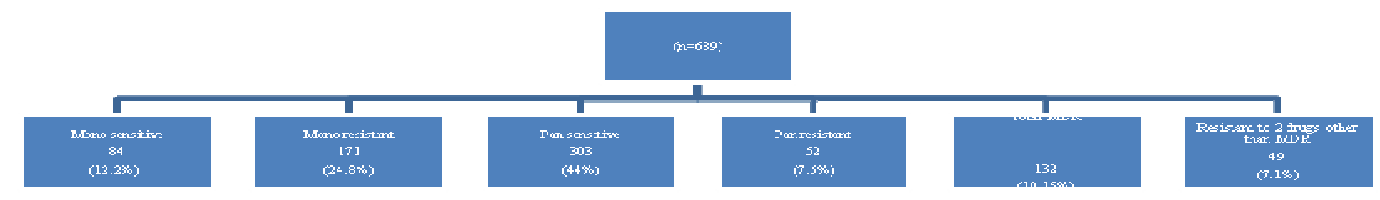

Figure 1: Sensitivity pattern of clinical MTB isolates $(n=689)$

\section{Discussion}

TB is a well known ancient disease. Five thousands new cases of TB are added daily, along with the loss of two lives due to TB every third minute. ${ }^{7}$ TB is the leading cause of mortality among infectious diseases worldwide; the majority of deaths occurring in the developing countries. ${ }^{8,9}$ TB may prove fatal for around 70 million people within next 2 decades if adequate measures are not timely adopted to prevent the spread. ${ }^{10}$

TB still remains one of the leading infectious diseases in the developing countries like
Pakistan. The key strategy to control the spread of this disease in community is early diagnosis, effective treatment and strict isolation of the infectious TB patients. MDR TB is becoming a real challenge in Pakistan with limited high-tech diagnostic facilities.

There has been a steady increasing trend in MTB resistance. By comparing our results with those of a study done at this institute 14 years ago by Karamat et al (1999) a rise of $6.2 \%$ resistance for INH and $4 \%$ for Rif was observed .11 Also, our study shows rising trend of mono resistance to Rif and INH, while surprisingly mono resistance to ETH 
and STR has significantly decreased. ${ }^{11}$ The reason for declining trend of resistance against STR may be due to the limited use of this drug nowadays.

In comparison to another study done at this institute by Ikram A et al (2008) resistance to one or more first line drugs has increased $8 \%$ within 7 years, while percentage of pansensitive isolates has decreased by $8.4 \% .^{12}$ Our results are also quite comparable with those of an Iranian study by Mirasaeidi et al (2007), which shows mono resistance to INH as $11 \%$, RIF $1.5 \%$ and ETH $1.5 \%{ }^{13}$ However, our isolates are less resistant to STR than Iranian study (12\%). ${ }^{13}$ Overall resistance to individual drugs is higher in our results with respect to another national study by Iqbal et al (2012) except for RIF; 4.7\% lesser resistant. ${ }^{14}$

With comparison to a study by Amjad A et al (2012), within 3 years number of mono resistant as well as pan-resistant ( $\mathrm{p}$-value = 0.221 ) cases increased from $18.2 \%$ to $24.8 \%$ and from $1.6 \%$ to $7.5 \%$, respectively. ${ }^{15}$ Regionally, in Bangladesh a study by Mottalib et al (2011) shows less overall resistance to individual drugs than our results. ${ }^{16}$ An Indian study by Sethi et al (2012) reported 16.6\% MDR-TB isolates with more cases of monoresistance to RIF in comparison to $19.16 \%$ MDR-TB (p-value $=0.312$ ) with more cases of mono-resistance to INH in our study. ${ }^{17}$

Out et al (2013) in a Nigerian study indicated much higher resistance to first line drugs as compared to our study. ${ }^{18}$ Similarly, a very high percentage $(58.4 \%)$ of MDR isolates and increased individual resistance to INH and RIF was reported by Green et al (2010) in a South African study. ${ }^{19}$

As expected in all laboratory based studies, the frequency of anti-TB drug resistance was higher as compared to general population, because most of the specimens were received from hospitalized patients or from complicated cases. Keeping in view the rising trend of resistance, there is an urgent need for a nationwide drug resistance surveillance program for assessing the actual prevalence of drug resistance within the country. Proper anti-TB drug management is an essential component for success of DOTS programme. ${ }^{20}$ Strict inventory management, efficient drug supply systems, quality assured drugs and appropriate prescription of anti-TB drugs are pivotal steps for TB control.

\section{Conclusion}

The ever increasing resistance to first line anti-TB drugs necessitates proper DST in order to have base line data to formulate effective anti-TB drug policy for guidance and accordingly patient treatment.

\section{References}

1. Sharam, S.K., Guleria, R., Jain, D., ehawla, T.C., Saha, P. and Mohan, A. et al. (1996) "Effect of additional oral ofloxacin administration in the treatment of multi-drug resistant tuberculosis." Indian J Chest Dis Allied Sci, 38: 73-9.

2. Grang, J.M. and Zumla, A. (2002) "The global emergency of tuberculosis: what is the cause?" J R Soc Health, 122: 78-81.

3. World Health Organization (2012) Global tuberculosis report. [Online] Available from:

http://apps.who.int/iris/bitstream/10665/7 5938/1/9789241564502_eng.pdf [Accessed: 13th May 2013].

4. NTP Pakistan annual report (2011) "Up scaling tuberculosis care and control." [Online] Available from: http://ntp.gov.pk/uploads/ntp_1369807897 _ANNUAL_REPORT_2011.pdf [Accessed: $10^{\text {th }}$ May 2013].

5. Kent, P.T. and Kubica, G.P. (1985) "Public health mycobacteriology, a guide for the level III laboratory." Centers for Disease Control, Development of Health and Human Services, Atlanta, Ga.

Tahir Ghafoor, Aamer Ikram, Shahid Ahmad Abbassi, Irfan Ali Mirza, Aamir Hussain, Inam ullah Khan and Jamal Ahmad (2014), Journal of Virology \& Microbiology, DOI: 10.5171/2014. 385616 


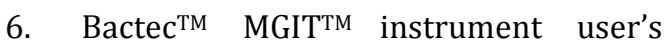
manual 2010/05, document number MA0117, catalog number 445876.

7. Tuberculosis Research Centre (2003) "The trends in initial drug resistance over three decades in a rural community in South India." Indian J Tuberc, 50: 75-86.

8. Rajeswari, R., Balasubramanian, R., Muniyandi, M., Geetharamani, S., Thresa, X. and Venkatesan, P. (1999) "Socioeconomic impact of tuberculosis on patients and family in India." Int J Tuberc Lung Dis, 3: 869-77.

9. Khuwaja, A.K. and Mobeen, N. (2005) "Knowledge about tuberculosis among patients attending family practice clinics in Karachi." JLUMHS, 4(2): 44-7.

10. Giri, P.K. and Khuller, G.K. (2008) "Is Intranasal Vaccination a Feasible Solution for Tuberculosis?" Expert Rev Vaccines, 7:134156.

11. Karamat, A.K., Rafi, S. and Abbasi, S.A. (1999) "Drug Resistance in Mycobacterium tuberculosis: A Four Years Experience." J Pak Med Assoc, 49:262-5.

12. Ikram, A., Ali, S., Ali, W. and Wiqar, M.A. (2008) "Latest Pattern of Multi-Drug Resistant Tuberculosis in Pakistan." Inf Dis J Pak, 17(1): 14-7.

13. Mirsaeidi, M.S., Tabarsi, P., Farnia, P., Ebrahimi, G., Morris, M.W. and Masjedi, M.R. et al. (2007) "Trends of drug resistant Mycobacterium tuberculosis in a tertiary tuberculosis center in Iran." Saudi Med J, 28(4):544-50.

14. Iqbal, R., Shabbir, I., Munir, k. and Qadeer, E. (2012) "The first and second line Anti TB drug resistance pattern in Lahore." PakJ Med Res, 51(1):1-4.
15. Amjad, A., Satti, A., Farwa, U. and Abbassi, S.A. (2012) "Rising trend of multidrug resistant tuberculosis: A threat to community." GJMS, 10(1):32-6.

16. Mottalib, A., Hossain, M., Khalil, I., Islam, S. and Hossain, A. (2011) "Drugs susceptibility pattern of Mycobacterium tuberculosis isolates against conventional anti-tuberculosis drugs in Dhaka, Bangladesh." Saudi Med J, 32(5): 484-8.

17. Sethi, S., Biswal, M., Chatterjee, S.S., Mewara, A., Gupta, D. and Kumar, S. et al. (2012) "Susceptibility pattern among pulmonary and extra pulmonary isolates of Mycobacterium tuberculosis in Northern India." Afr J Microbiol Res, 6(15): 3696-9.

18. Out, A., Umoh, V., Habib, A., Ameh, S., Lawson, L. and Ansa, V. (2013) "Drug resistance among pulmonary tuberculosis patients in Calabar, Nigeria." Hindawi Publishing Corporate. [Online] Available from:

http://www.hindawi.com/journals/pm/201 3/235190/. [Accessed: 6th Dec 2013].

19. Green, E., Obi, C.L., Nchabeleng, M., deVilliers, B.E., Sein, P.P. and Letsoalo, T. (2010) "Drug susceptibility patterns of Mycobacterium tuberculosis in Mpumalanga province, South Africa: Possible guiding design of retreatment regimen." J Health Popul Nutr, 28(1): 7-13.

20. Shah, S.A., Kristensen, S., Memon, M.A., White, H.L. and Vermund, S.H. (2007) "Syndromic management training for nonformal care providers in Pakistan improves quality of care for sexually transmitted diseases: A randomized clinical trial." Southeast Asian J Trop Med Public Health, 38(4):737-48.

Tahir Ghafoor, Aamer Ikram, Shahid Ahmad Abbassi, Irfan Ali Mirza, Aamir Hussain, Inam ullah Khan and Jamal Ahmad (2014), Journal of Virology \& Microbiology, DOI: 10.5171/2014. 385616 